\title{
Amnesia after a discrete basal forebrain lesion
}

\author{
Kazuo Abe, Maki Inokawa, Asako Kashiwagi, Takehiko Yanagihara
}

\begin{abstract}
Destructive lesions of the basal forebrain are often associated with memory impairment and this structure is thought to contribute to memory function by providing a cholinergic input to critical structures associated with memory such as the hippocampus and amygdala. In previously reported cases of amnesia associated with damage in the basal forebrain, multiple neuroanatomical regions were damaged, and the critical lesion responsible for amnesia has not been identified clearly. We report a patient who developed primarily anterograde amnesia after clipping of an unruptured anterior communicating artery aneurysm. Postoperative magnetic resonance imaging showed a discrete lesion, centring in the right diagonal band of Broca and including the anterior hypothalamus, septal nucleus, lamina terminalis, and paraterminal gyrus, and an indiscrete patchy lesion in the corresponding area on the opposite side. The nucleus basalis of Meynert was minimally affected and the diencephalon was not damaged. Single photon emission computed tomography showed marked hypoperfusion in the midline frontobasal region corresponding to the MRI lesion and hypoperfusion in the hippocampus bilaterally. It is concluded that disconnection of the pathway between the diagonal band of Broca and the hippocampus contributed to memory impairment.

(F Neurol Neurosurg Psychiatry 1998;65:126-130)
\end{abstract}

Keywords: diagonal band of Broca; amnesia; time tagging

The amnesic syndrome has been found in patients with relatively discrete lesions in several cerebral sites including $(a)$ the medial temporal lobes including the hippocampus, $(b)$ median thalamic nuclei, $(c)$ anterior thalamic nuclei, $(d)$ basal forebrain, (e) fornix, $(f)$ mammillary bodies, and $(g)$ retrosplenial cortex. ${ }^{1-3}$ The basal forebrain has been considered to contribute to memory function by way of the pathways between nuclei of the diagonal band or the septal nucleus and the hippocampal region, thus providing a cholinergic input to the hippocampus. ${ }^{1-4}$ In most previously reported cases of amnesia ${ }^{4-14}$ associated with basal forebrain lesions, multiple neuroanatomically identified areas were damaged, and it has been difficult to identify the minimal lesion necessary to produce an amnesic syndrome except for patients in whom basal forebrain lesions were relatively well circumscribed. ${ }^{4}$ We describe a patient who developed anterograde amnesia as the result of a discrete lesion in the basal forebrain after clipping of an unruptured anterior communicating artery aneurysm.

\section{Case report}

A 61 year old right handed high school teacher, who had taught science for more than 35 years and had had good memory function, experienced a sudden onset of weakness in the right upper and lower limbs in July 1993, but recovered the next day. He was admitted to a hospital elsewhere and underwent MRI, which showed a small haemorrhage in the left external capsule. He underwent cerebral angiography, which showed an aneurysm $5 \mathrm{~mm}$ in diameter in the anterior communicating artery. $\mathrm{He}$ underwent surgical clipping of the aneurysm one month later. Immediately after the surgery, he developed disorientation and agitation. Although he became alert and oriented to person and place one month after the operation, he remained disoriented to date. $\mathrm{He}$ apparently had memory impairment at that time, but the details are not known to us. $\mathrm{He}$ had no motor or sensory deficits.

$\mathrm{He}$ visited the neurology outpatient service of the Osaka University Hospital four months after the operation. He was alert and did not have any focal neurological deficits. He did not seem to appreciate the graveness of his memory impairment. His language comprehension and expression were intact. He could repeat seven digits and perform serial subtraction without difficulty. He could learn five objects but could recall only one of them after five minutes. During the interview, his recall was aided by cuing. Recall of his remote personal history was relatively preserved but the dates were mixed up, and he mistook recent personal episodes as remote ones. He did not have confabulation and remained silent when he could not answer a question. An EEG showed no abnormality.

\section{NEUROPSYCHOLOGICAL ASSESSMENT}

Formal neuropsychological testing was performed several times over 36 months, and two series are presented here, the one at the initial 
Table 1 Neuropsychological assessment

\begin{tabular}{lll}
\hline & $1994 / 2$ & $1996 / 2$ \\
\hline MMSE & 25 & 27 \\
WAIS & 108 & 112 \\
VIQ & 103 & 116 \\
PIQ & 0 & 0 \\
Cancellation tasks (errors) & 36 & 36 \\
Rey-Osterrieth-copy & 98 & 98 \\
Token test (\%) & 50 & 50 \\
Boston naming & 7 & 7 \\
Digit span & 5 & 5 \\
$\quad$ Forward & & \\
$\quad$ Backward & 6 & 6 \\
Wisconsin card sorting test & 6 & 3 \\
Category & $30 ”$ & $31 ”$ \\
Perseveration errors & $55 ”$ & $48 ”$ \\
Trail making test A & & \\
Word fluency test B & 18 & 19 \\
$\quad$ Category & 16 & 18 \\
$\quad$ Letter head & & \\
\hline
\end{tabular}

Table 2 Memory assessment

\begin{tabular}{|c|c|c|}
\hline & $1994 / 2$ & $1996 / 2$ \\
\hline \multicolumn{3}{|l|}{ WMS-R: } \\
\hline Verbal memory & 69 & 82 \\
\hline Nonverbal memory & 104 & 108 \\
\hline General memory & 77 & 90 \\
\hline Attention/concentration & 92 & 89 \\
\hline Delayed recall & 65 & 78 \\
\hline \multicolumn{3}{|c|}{ Rey auditory verbal learning: } \\
\hline Maximum recall & 9 & 11 \\
\hline After interference & 0 & 2 \\
\hline Recognition (/50) & 40 & 44 \\
\hline \multicolumn{3}{|l|}{ Work fluency (/min): } \\
\hline Letter head & 13 & 14 \\
\hline Category & 12 & 14 \\
\hline \multicolumn{3}{|l|}{ Benton visual retention: } \\
\hline \multicolumn{3}{|l|}{ Immediate } \\
\hline Errors & 6 & 8 \\
\hline Corrects & 5 & 6 \\
\hline \multicolumn{3}{|l|}{ Delay } \\
\hline Errors & 13 & 8 \\
\hline Corrects & 2 & 4 \\
\hline \multicolumn{3}{|c|}{ Paired verbal association ( 3 trials): } \\
\hline Related & $4-6-8$ & $5-7-8$ \\
\hline Unrelated & $0-0-0$ & $1-2-2$ \\
\hline Tower of Toronto (index) & 18 & 16 \\
\hline \multicolumn{3}{|l|}{ Remote recall (\%): } \\
\hline \multicolumn{3}{|l|}{ Items before operation } \\
\hline Recall & 94 & 98 \\
\hline Recognition & 100 & 100 \\
\hline \multicolumn{3}{|l|}{ Items after operation } \\
\hline Recall & 3 & 8 \\
\hline Recognition & 8 & 15 \\
\hline \multicolumn{3}{|c|}{ Remote autobiographical recall: } \\
\hline Recall & 70 & 80 \\
\hline Recognition & 100 & 100 \\
\hline
\end{tabular}

evaluation in February 1994 and another 24 months later (tables 1 and 2).

First assessment

At the time of the first evaluation in February 1994, he was alert, cooperative, and oriented to person and place. His verbal IQ and non-verbal IQ fell within the normal range. ${ }^{15}$ The results of the Wisconsin card sorting test ${ }^{16}$ trail making test, ${ }^{17}$ word fluency, ${ }^{18}$ and go/no go task $^{17}$ to measure the frontal lobe function were normal. Performance on all other neuropsychological measures fell within the normal range.

He obtained a verbal memory quotient of 69 , whereas the non-verbal memory quotient was 104 on the Wechsler memory scale-revised (WMS-R). ${ }^{19} \mathrm{He}$ clearly showed mismatch between verbal and non-verbal memory function. He was able to repeat seven digits forward and five digits backwards, indicating an intact attention span. ${ }^{17}{ }^{18}$ Although he could learn
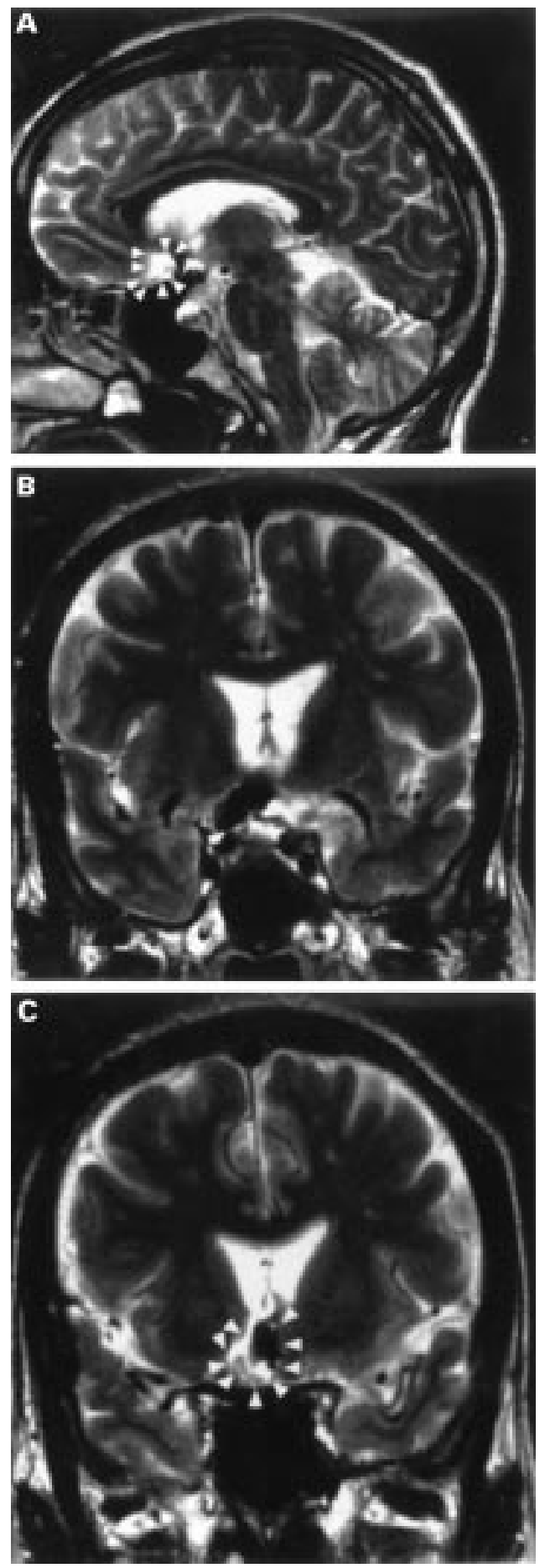

Figure 1 Magnetic resonance image in February 1996, 30 months after surgery. $A$ sagittal image $(A)$ and two coronal images $(B, C)$ on the $T 2$ weighted pulse sequence show a discrete lesion located in the right basal forebrain including the diagonal band of Broca, anterior hypothalamus, and the lamina terminalis. Imaging was carried out with a 1.5 Tesla superconducting system with a spin echo method. The T2 weighted pulse sequence was carried out by using the repetition time (TR) of $5000 \mathrm{~ms}$ and the echoing time (TE) of $130 \mathrm{~ms}$.

four out of 10 easy paired associates, he could not learn any of the difficult word pairs. He was impaired on a word list learning task, recalling no more than nine out of 15 words. Although his recall of the word list dropped to zero after performing an interfering word list, he had recognition of 40 out of 50 words presented in a 


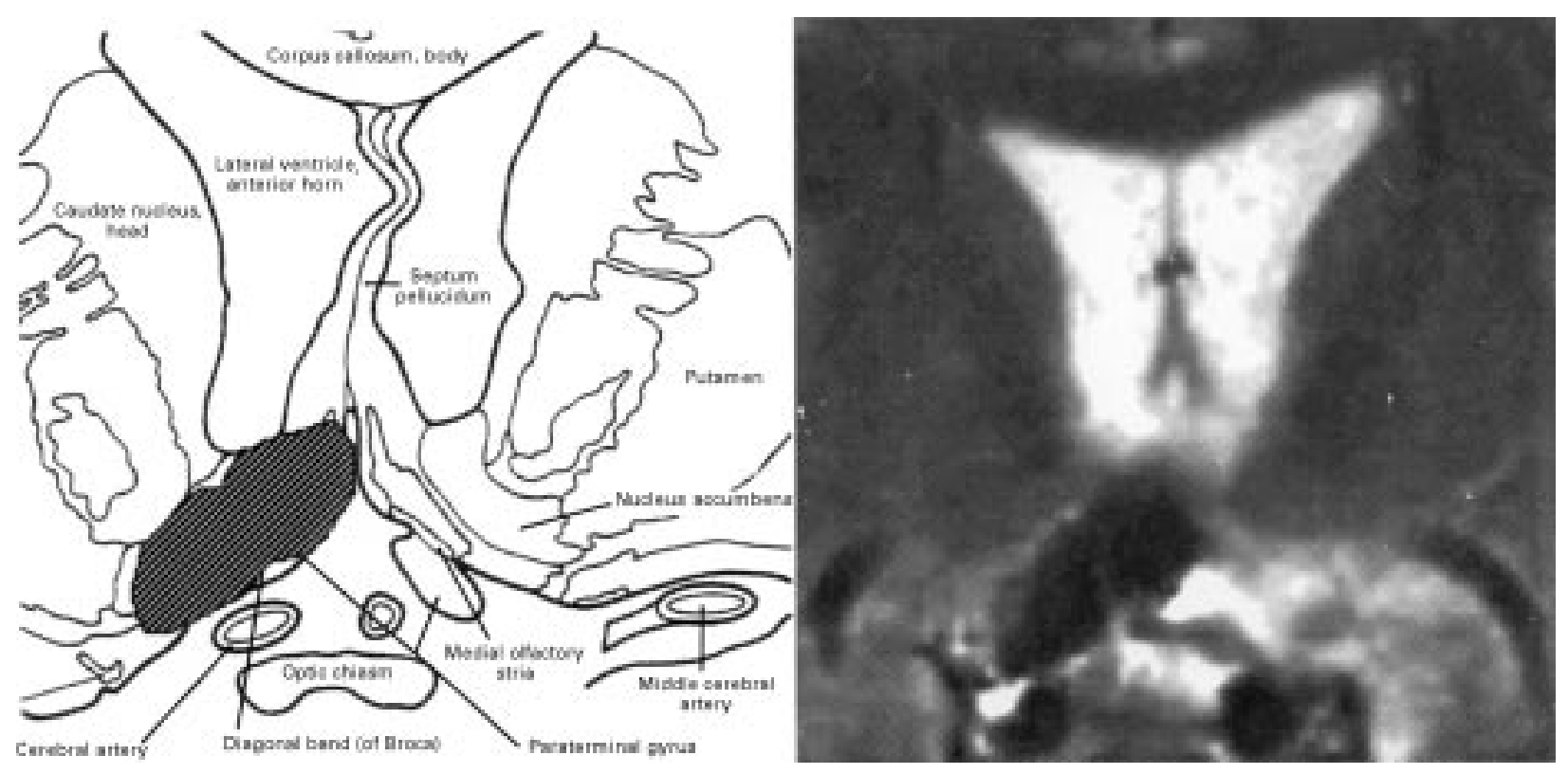

Figure 2 Coronal section from the Haines neuroanatomical atlas indicates that the lesion was in the basal forebrain including the diagonal band of Broca, anterior hypothalamus, and the lamina terminalis.

yes/no recognition paradigm. ${ }^{20}$ His recent nonverbal memory was relatively preserved. On the visual reproduction portion of the WMS-R, he obtained a non-verbal memory quotient of 104, which fell within the normal range.

A

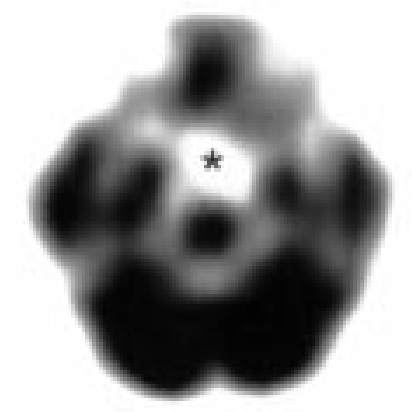

R

B

Immediate and delayed recall of the Benton visual retention test ${ }^{21}$ were moderately impaired. Memory for past public events was examined using the method described by Fukatsu et $a l^{12}$ and was within the normal range for the 1950 s to the $1980 \mathrm{~s}$. He showed a limited period of retrograde amnesia for events before his operation. With the help of his family, a list for personal autobiographical memory was constructed with questions about significant personal events (for example, marriage, death, children's accomplishments). Although he could recall almost all events, he could not tag these events in sequence.

\section{Follow up assessment}

He was assessed in February 1996, 30 months after surgery. He showed mild improvement in memory performance, predominantly in nonverbal memory (tables 1 and 2). Immediate and delayed recall of the Benton visual retention test fell within the normal range. Although his verbal memory quotient on the WMS-R increased from 69 to 82, it remained impaired compared with his non-verbal memory quotient of 108 . He achieved normal maximal learning of a word list learning task, but he continued to show severely impaired delayed recall. On memory for past public events and personal autobiography, he could recall each event fairly well. However, he could not arrange these events in sequence and mistook the latest event for one having occurred years before.

R

Figure 3 Single photon emission computed tomography using ${ }^{99 m} T c$ - d, l-hexamethylpropyleneamine oxime ${ }^{99 m} T_{c}$ HMPAO) 30 months after surgery. Scanning was carried out 15 minutes after intravenous injection of $740 \mathrm{mBq}$ $99 m T c-H M P A O$. An axial SPECT image parallel to the orbitomeatal line (left) shows a defect ${ }^{*}$ ) in the midline expanding to both sides corresponding to the lesion on $M R$ I. Another axial image parallel to the hippocampus (right) shows mild hypoperfusion bilaterally in the hippocampus (arrows) but slightly more reduction on the left side.
NEUROIMAGING EXAMINATION

Brain MRI with a 1.5 Tesla superconducting system (General Electric), disclosed a small discrete lesion in the basal forebrain slightly to the right of the midline in February 1996 (fig 1). Mapping of the lesion by superimposing on two schematic coronal sections from the Haines neuroanatomical atlas ${ }^{23}$ indicated that the lesion was in the basal forebrain including 
the diagonal band of Broca, septal nucleus, anterior hypothalamus, and the lamina terminalis (fig 2). There was an indiscrete patchy lesion in the corresponding area on the opposite side on the T2 weighted image (fig 2) but this lesion was identified on only one slice. The involvement of the nucleus basalis of Meynert was minimal and neither the medial temporal lobe nor diencephalon was damaged. The orbitofrontal and cingulate cortex were also spared. The appearance of the lesion on the right side indicated the presence of hemosiderin deposition. Single photon emission computed tomography (SPECT) using ${ }^{99 m} \mathrm{Tc}-\mathrm{d}, \mathrm{l}$-hexamethyl propyleneamine oxime showed an area of hypoperfusion, below $40 \%$ of the maximal isotope uptake in the cerebellum, in the midline frontobasal region expanding to both sides corresponding to the lesion on the MR image (fig 3). In addition, the isotope uptake in the hippocampus was below $50 \%$ of the maximal isotope uptake in the cerebellum bilaterally, but slightly more reduced on the left side (fig 3).

\section{Discussion}

In summary, our patient showed (a) anterograde amnesia, $(b)$ mild retrograde amnesia for both semantic and personal episodic information, (c) benefit from cuing, and (d) defects in the temporal tagging of information. The clinical presentation was similar to the previously reported cases of amnesia after damage to the basal forebrain and adjacent structures. $^{3}{ }^{14}$ As reviewed previously, ${ }^{24}$ those cases were mostly associated with a ruptured anterior communicating artery aneurysm. However, previously reported cases ${ }^{5-13}$ did not have such a discrete lesion in the basal forebrain as our patient, whose anterior communicating artery aneurysm was unruptured.

Our patient could learn test items in the memory evaluation batteries, but he had difficulty in recall and could not develop a proper time sequence for this information even when he could recall. This was striking and was different from memory impairment described in patients $\mathrm{HM}$ and $\mathrm{DRB}$, whose failures were in learning both types of information..$^{25}$ The availability of contextual memory is thought to depend on the recording of separate information that is composed of a perceived episode, computation, and recoding of the relation between multiple components, and time tagging of this information. Despite his ability to learn information correctly, our patient showed difficulty in his daily living. This may be due to the difficulty in forming time tagging of this information. Because cuing markedly improved recall and recognition of both anterograde and retrograde memories, his amnesia with preserved cuing may be due to a deficit in retrieval. This notion was supported by the clinical finding and the result of the Rey's auditory verbal learning test. ${ }^{20}$

It is difficult to ascertain the minimal lesion necessary to cause the amnesic syndrome associated with a basal forebrain lesion, because of the extent and variability of the previously reported lesions. There are only two patients in the literature who had a relatively well circumscribed lesion. ${ }^{4}{ }^{5}$ Damasio et $a l^{4}$ described a patient (case 1) who had haematomas in the left gyrus rectus and midline septal nuclei, who subsequently underwent surgical clipping of the ruptured left anterior cerebral artery aneurysm. The extent of the lesion was delineated by CT. Another patient described by Morris et $a \bar{l}$ had a low grade glioma near the posterior extent of the right gyrus rectus involving the lamina terminalis and developed severe amnesia postoperatively. Postoperative MRI showed a discrete lesion involving the right diagonal band of Broca, preoptic area, anterior hypothalamus, lamina terminalis, and paraterminal gyrus. There are some similarities and dissimilarities among these two cases and ours. Damasio et al found a mismatch between auditory-verbal and non-verbal memories as we did. On the other hand, the patient described by Morris et al had a lesion predominantly on the right side, but did not have such a mismatch. Our patient also had a lesion mostly to the right of the midline on MRI, but possessed an area of profound hypoperfusion in the midline frontobasal region expanding to both sides on SPECT. He showed better performance on the non-verbal memory examination than the verbal one. As the projection of the diagonal band to the hippocampus is predominantly ipsilateral, his lesion should have affected the projection to the right hippocampus. He showed complete right handedness by the handedness inventory ${ }^{27}$ and had no family history of left handedness or childhood events that might be associated with anomalous lateralisation of cognitive function. At the present time, there is no clear explanation for better performance on the non-verbal memory examination despite the more extensive lesion in the right basal forebrain on MRI examination. One possibility is that the left hippocampus was functionally more impaired in our patient, as the SPECT examination suggested. Another possibility is the presence of hemispheric asymmetry for retrieval of episodic memory. According to Tulving et al, there was increased blood flow in the right prefrontal cortex at the time of retrieval of episodic memory. ${ }^{28}$ They concluded that the left prefrontal cortex was more involved in retrieval of episodic memory. ${ }^{29}$ Although their finding was confined to the cerebral cortex, it is possible that the retrieval of information may involve the pathway from the basal forebrain to amygdala or hippocampus and then to the prefrontal cortex, and time tagging of information may be more reflected in verbal or episodic memory. Further case analyses would be necessary to confirm or rule out this possibility.

Summarising these three patients who had discrete lesions in the basal forebrain, the critical anatomical lesion may be confined to the nuclei of the diagonal band. Although other possibilities cannot be entirely dismissed, the primary underlying aetiology of amnesia resulting from a lesion in the basal forebrain thus seems to be disconnection of the critical bidirectional connections between the nuclei of 
the diagonal band and the hippocampus. This idea is supported by the result of SPECT showing a lesion in the basal forebrain and reduction of blood perfusion in the hippocampus bilaterally. It is also possible that amnesia caused by a lesion in the basal forebrain with interference of the medial temporal function may develop impairment in the time tagging of information.

We obtained written informed consent to report on this patient.

1 Aaral DG. Memory anatomical organisation of candidate brain regions. In: Mountcastle FP, Geiger SR, eds. Handbook of physiology. Section 1. The nervous system. Vol V. Higher function of brain. Part I. Bethesd 2 Aggleton JP. Anatomy of memory. In: Yanagihara T, Petersen RC, ed. Memory disorders: research and
practice. New York: Marcel Dekker, 1991:23-62.

3 Von Cramon DY, Hebel N, Schuri U. A contribution to the anatomical basis of thalamic amnesia. Brain 1985;108;9931008.

4 Damasio AR, Graff-Radford NR, Eslinger PJ, et al. Amnesia following basal forebrain lesions. Arch Neurol 1985;42:263 71.

5 Morris MK, Bowers D, Chatteriee A, et al. Amnesia following a discrete basal forebrain lesion. Brain 1992;115:182747

6 Lindqvist G, Norlen G. Korsakoff's syndrome after operation on ruptured aneurysm of the anterior communicating artery. Acta Psychiatr Scand 1966;42:24-36.

7 Talland GA, Sweet WH, Ballantine HT. Amnestic syndrome with anterior communicating artery aneurysm. 7 Nerv with anterior communicating

8 Logue V, Durnard M, Pratt RTC, et al. The quality of survival after rupture of an anterior communicating artery vival after rupture of an anterior communica

9 Gade A. Amnesia after operations on aneurysms of the anterior communicating artery. Surg Neurol 1982;18:46-9.

10 Volpe BT, Hirst W. Amnesia following the rupture and repair of an anterior communicating artery aneurysm. $f$ Neurol Neurosurg Psychiatry 1983;46:704-9.

11 Alexander MP, Freedman M. Amnesia after rupture of an anterior communicating artery aneurysm. Neurology 1984 34:752-7.
12 Parkin AJ, Leng NRC, Stanhope N, et al. Memory impairment following ruptured aneurysm of the anterior communicating artery. Brain Cogn 1988;7:231-43.

13 Phillips S, Sangalang V, Sterns G. Basal forebrain infarction. A clinicopathologic correlation. Arch Neurol 1989;44: 1134-8.

14 Vilkki J. Amnestic syndromes after surgery of anterior communicating artery aneurysms. Cortex 1985;21:431-44

15 Wechsler D. Wechsler adult intelligence scale - revised. Anglicised revision. Sidcup: Psychological Corporation, 1986.

16 Milner B. Some effects of frontal lobectomy in man. In: Warren JM, Akert $\mathrm{K}$, ed. The frontal granular cortex and behavior. New York: McGraw - Hill, 1964:313-34.

17 Lezak MD. Neuropsychological assessment. 3rd ed. Oxford: Oxford University Press, 1995.

18 Hirono N, Yamadori A, Kameyama M, et al. Spinocerebellar degeneration (SCD): cognitive disturbances. Acta Neurol Scand 1991;84:226-30,

19 Wechsler D. Wechsler memory scale - revised manual. San Antonio, TX: The Psychological Corporation, 1991.

20 Taylor LB. Localization of cerebral lesions by psychological testing. Clin Neurosurg 1969;16:269-87.

21 Benton AL, Spreen O. Visual memory test: the simulation of mental incompetence. Arch Gen Psychiatry 1961;4:79-83.

22 Fukatsu R, Fujii T, Sati M, et al. Influence of age on long term memory. Clin Neurol 1994;34:777-81. (In Japanese.)

23 Haines DE. Neuroanatomy: an atlas of structures, sections, and systems. 4th ed. Baltimore: Williams and Wilkins, 1995.

24 Yanagihara T. Memory disorder in cerebral vascular disease. In: Yanagihara T, Petersen RC, ed. Memory disorders: research and clinical practice. New York: Marcel Dekker, 1991:197-226.

25 Scoville WB, Milner B. Loss of recent memory after bilateral hippocampal lesions. F Neurol Neurosurg Psychiatry 1957; 20:11-21.

26 Damasio AR, Eslinger PJ, Damasio H, et al. Multimodal amnesic syndrome following bilateral temporal and basal forebrain damage. Arch Neurol 1985;42:252-9.

27 Briggs GG, Nebes RD. Patterns of hand preference in a student population. Cortex 1975;11:230-8.

28 Tulving E, Kapur S, Markowitsch HJ, et al. Neuroanatomical correlates of retrieval in episodic memory: auditory sentence recognition. Proc Natl Acad Sci 1994;91:2012-5.

29 Tulving E, Kapur S, Fergus I, et al. Hemispheric encoding/ retrieval asymmetry in episodic memory: positron emission tomography findings. Proc Natl Acad Sci 1994;91:2016-20. 